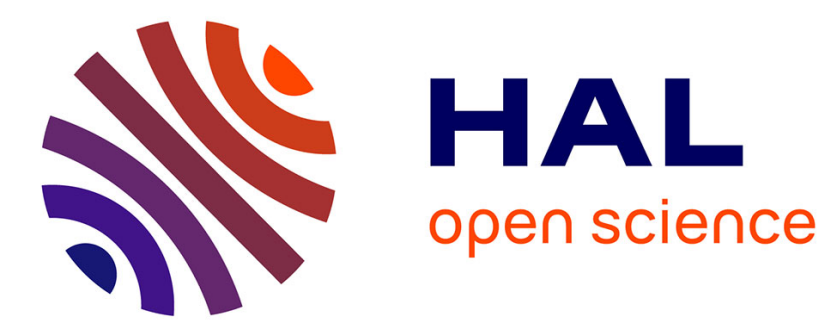

\title{
Impact of travelers information level on disturbed transit networks: a multiagent simulation
}

Mahdi Zargayouna, Amine Othman, Gérard Scemama, Besma Zeddini

\section{To cite this version:}

Mahdi Zargayouna, Amine Othman, Gérard Scemama, Besma Zeddini. Impact of travelers information level on disturbed transit networks: a multiagent simulation. IEEE Intelligent Transportation Systems Conference, Sep 2015, LAS PALMAS, Spain. pp. 2889-2894. hal-01217377

\section{HAL Id: hal-01217377 \\ https://hal.science/hal-01217377}

Submitted on 19 Oct 2015

HAL is a multi-disciplinary open access archive for the deposit and dissemination of scientific research documents, whether they are published or not. The documents may come from teaching and research institutions in France or abroad, or from public or private research centers.
L'archive ouverte pluridisciplinaire HAL, est destinée au dépôt et à la diffusion de documents scientifiques de niveau recherche, publiés ou non, émanant des établissements d'enseignement et de recherche français ou étrangers, des laboratoires publics ou privés. 


\title{
Impact of travelers information level on disturbed transit networks: a multiagent simulation
}

\author{
Mahdi Zargayouna $^{a} \quad$ Amine Othman $^{a} \quad$ Gérard Scemama $^{a} \quad$ Besma Zeddini $^{b}$ \\ ${ }^{a}$ Université Paris Est, IFSTTAR, GRETTIA \\ 10-14, boulevard Newton, \\ 77447 Champs sur Marne, France. \\ ${ }^{b}$ Quartz, EISTI \\ Avenue du Parc, \\ 95000 Cergy Pontoise, France. \\ \{hamza-mahdi.zargayouna, amine.othman, gerard.scemama@ifsttar.fr\}@ifsttar.fr \\ besma.zeddini@eisti.eu
}

\begin{abstract}
With the generalization of real-time traveler information, the behavior of modern transport networks becomes harder to analyze and to predict. It is now critical to develop simulation tools for mobility policies makers, taking into account this new information environment. Information is now individualized, and the interaction of a population of individually guided travelers have to be taken into account in the simulations. Multiagent simulation is proving to be a powerful model for analyzing transport activities. Indeed, autonomous agents provide an appropriate basis for modeling heterogeneous systems and environment-based systems. In this paper, we present a multiagent simulation model to measure the impact of information provision on the quality of passengers travels. This impact is measured by simulating different scenarios in function of the percentage of connected travelers, represented as agents. These simulated scenarios are analyzed following their impact on the average travel times of the travelers. Results show that the number of connected travelers has a positive impact on overall travel times up until a certain threshold before becoming negative.
\end{abstract}

\section{INTRODUCTION}

Transport systems are more and more complex and they have to evolve to integrate more connected entities (mobile devices, connected vehicles, etc). Indeed, we can now provide optimal routes for the travelers but we are also able to update these routes in real time based on new network status (congestions, accidents, bus down, etc). Giving information to the traffic network users is generally good and allows the improvement of the global network traffic flows. However, without control, the massive spread of information via billboards, radio announcements and individual guidance may have perverse effects and create new traffic jams. Indeed, with this generalization of real-time traveler information, the behavior of modern transport networks becomes harder to analyze and to predict. It is then important to observe these effects to consider the proper methods to deal with them.

Indeed, nowadays traveler information systems have great benefits (alleviate congestion, reducing waiting/travel time), however they might have side effects. [3] listed three phenomena that can be observed after the use of advanced traveler information systems:

i Over-saturation: when travelers are confronted with too much information, they tend to ignore the information and try to solve the problem by their own means.

ii Concentration: when traveler with same preferences are given the same routes, congestion is created.

iii Over-reaction: if too many travelers respond to a provided alternative, congestion may be transferred from the original area to the alternative one.

In this paper, we evaluate by simulation the benefits and limits of real-time information provision to travelers, especially in disturbed situations. To this end, we use the multiagent paradigm, which is proven to be a powerful model to design and implement transportation applications. Indeed, the multiagent approach deals with systems consisting of many physically and/or logically distributed interacting components that possess some level of autonomy. These components are able to perceive their environment and also react to changes in that environment in accordance to their goals. That is why the multiagent approach is adapted to the transportation domain since it facilitates an approach by analogy in a domain where the objective is the management of distributed entities. The authors in [1] list several reasons for the privileged use of multiagent systems in these applications, such as the natural and intuitive problem solving, the ability of autonomous agents for the modeling of heterogeneous systems, the ability to capture complex constraints connecting all problem-solving phases, etc. This approach is particularly relevant for the simulation 
of travelers mobility since the objective is to take into account human behaviors that interact in a complex, dynamic and open environment [4]. Indeed, the concept of an agent is well suited for the representation of travelers in transit or road traffic scenarios. They are autonomous entities which are situated in an environment, adapt their behaviors to the dynamics they perceive and interact with others agents in order to achieve specific goals.

The remainder of this paper is structured as follows. In section II, we discuss the choice of the simulation platform and previous proposals for the impact of information on travelers. In section III, we detail the simulator design: its parameters and data, the behaviors of the agents in the system, the path planning and the representation of the network. In section IV, we describe our experiments before to conclude and describe some further work we are conducting.

\section{StATE OF THE ART}

To design and implement a multiagent simulator, it is possible to develop an application directly in a host programming language. However, it is often faster, more useful and more efficient to ground the simulator on an existent multiagent simulation platform. In the context of transportation applications, one main choice criterion for the simulation platform is its ability to create geospatial agent-based models, i.e. its ability to integrate and process geographic data. Based on this criterion, we haven't considered several popular multiagent platforms such as Jade [2], Mason [10] and Madkit [7], for which it is difficult to integrate GIS capabilities.

Swarm [13] is a simulation platform that has a library allowing for the loading of layers of GIS data. However, it does not provide spatial primitives nor gives the possibility to store the resulted environment [16]. Netlogo allows to import and export GIS data and provides some basic geometrical operations, but not the more advanced spatial analysis operations. Gama [16] provides an environment for building spatially explicit agent-based simulations, while Repast Simphony [17] integrates a GIS library (Geotools), and provides additional GIS services (network modeling as a graph, computation of shortest paths, visualization and management of $2 \mathrm{D}$ and $3 \mathrm{D}$ data, etc.). Between all the available simulation platforms that fit with our requirements, the Repast Simphony platform is the most mature one and more importantly, it integrates several Api to deploy simulators over several hosts $^{1}$. This is why our simulator SM4T is based on this platform.

Besides, there exists several multiagent simulators for travelers mobility. For instance, MATSim [11] is a widely known platform for mobility micro-simulation. However, the mobile entities in MATSim are passive and their state is modified by central modules, which limits its flexibility and its ability to integrate new types of (proactive)

\footnotetext{
${ }^{1}$ The distribution of our simulator over several hosts is one of our ongoing works.
}

agents. Transims [14] simulates multimodal movements and evaluates impacts of policy changes in traffic or demographic characteristics while Miro [5] reproduces the urban dynamics of a French city and proposes a prototype of multiagent simulation that is able to test planning scenarios and to specify individuals' behaviors. AgentPolis [9] is also a multiagent platform for multimodal transportation. However, none of these proposals considers connected travelers. In the proposal described in this paper, connected travelers routes are continuously monitored and alternatives are proposed to them in case of disturbances.

The impact of information on travelers choices and transport networks has also been investigated in the literature. In [8], the authors present an analytic framework to evaluate path choices and travel time benefits resulting from real-time information. The disturbances are not taken into account in this model. In [6], the author describe a case study implementing a simple agent-based route choice decision model within a microscopic traffic simulation tool. This study is circumscribed to drivers. This is also the case for [18] address a basic two-route scenario with different types of information and study the impact of it using simulations. Like in our approach, the road users are modeled as agents. As we can see, there exists several studies on the assessment of the impact of trafic information on travelers. However, to the best of our knowledge, there exists no multiagent simulation that was dedicated to the assessment of the impact of both fixed and mobile information on transit passengers, including connected travelers, especially with the consideration of disturbances.

\section{Multiagent Simulator}

We use the SM4T simulator [19] for the simulation of travels on an urban network. SM4T is a multiagent multimodal simulation platform that allows for the individual monitoring of travelers on an urban multimodal network. However, it has no representation of the information flows in the network. Hence, we have removed the road network travels from SM4T and enriched it with traveler information capabilities, both at the stops and with personal information. Here follows a description of the simulation as we are using it.

The simulator represents itinerary planers, passengers, public transport vehicles and information means in a micro-level and simulate their dynamic movements. To this end, it needs input data and some additional parameters.

\section{A. Data and Parameters}

The workflow of Figure 1 details one simulation execution and structures the remainder of the presentation. The simulation starts with the loading of the parameters (simulation duration, number of agents of each type, the default speeds of each agent type, etc.). Then, the main 


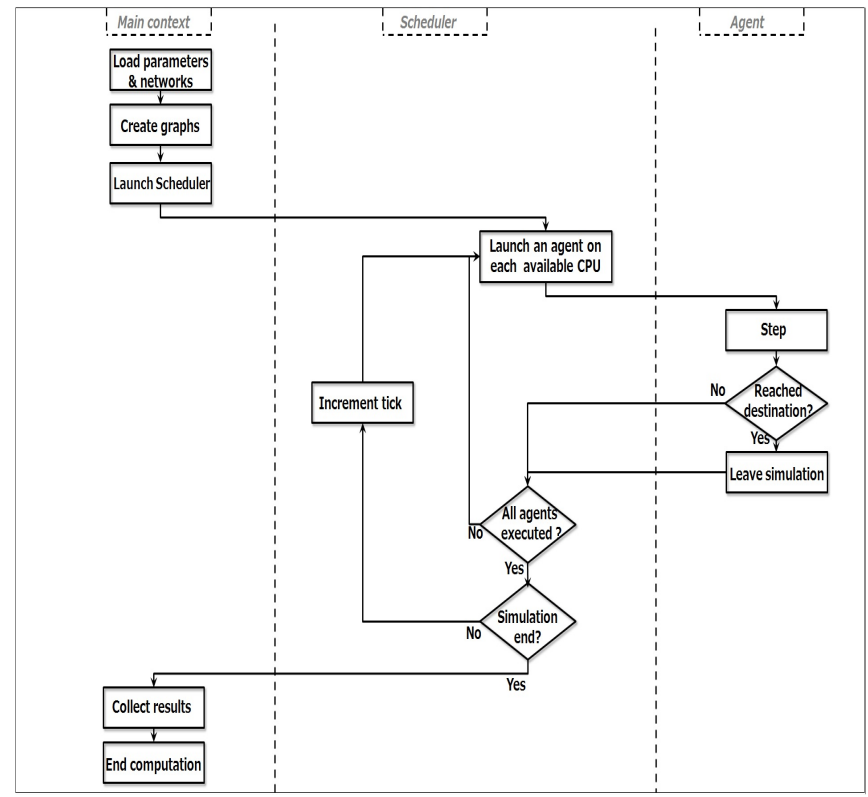

Fig. 1. Workflow of the simulation

context program creates the logical graphs (described in section III-C) and launches the scheduler. The scheduler is responsible of the synchronization of execution of the agents per tick of simulated time. We have defined a scheduler that launches the active agents in parallel over all the available CPUs. When launched, each agent executes a step method. The behavior of each agent type is described in section III-B. Finally, when the simulation duration is reached, the results are reported and the simulation ends.

1) Data: The input data (xml files) of the simulator are:

- the public transport network,

- the transfer mapping,

- the timetables of the public transport vehicles,

- the pedestrian network,

- the travel patterns,

- the travelers profiles.

A public transport network is composed of transport lines, each of them composed of a set of itineraries. An itinerary is composed of a sequence of edges. Each edge has a tracing in the form of a sequence of pairs $\langle$ longitude, latitude $\rangle$, and is composed of an origin node and a destination node. Finally, every node is defined by its name and coordinates. The transfer mapping is a table informing about the stops of the network for which a transfer by foot is possible and the road transport nodes that are reachable from the stops. The timetables of the vehicles are composed of a set of missions. Each mission corresponds to a specific itinerary and describes the path of a vehicle and the corresponding visit times. Each timetable is then a sequence of pairs $\langle$ stop, time $\rangle$. The pedestrian network is a subset of the road network in which pedestrians can move, and is undirected (pedestrians don't have to obey to the one-way limitations). A travel pattern clusters the considered geographic region in zones and describes the number of persons asking to leave or to join each region. The travelers profiles define the properties and preferences of the travelers. When passengers are connected, his preferences also define the accepted time gap between their computed itinerary and their real situation, before asking for a new up-to-date itinerary.

2) Parameters:

a) Simulation duration: Two values define the duration of a simulation run. The first is an interval defining the first and last date that are simulated (noted $\tau^{-}$and $\tau^{+}$respectively). In the absence of these parameters, we use respectively the values associated to the maximum date and minimum date in the timetables of the public transport vehicles. The second value that has to be defined is the number of discrete ticks of time that the simulation will execute before terminating (noted $\delta$ henceforth). At each tick, all the agents are activated for a particular action defined by their behavior.

b) Agents speeds: The speeds of the agents are defined in two ways:

- Public transport vehicles: based on the input data, they are inferred from the $\langle$ stop, time $\rangle$ pairs.

- Default: the simulator user defines speeds for pedestrian and public transport vehicles as a parameter. The user-defined public transport speed is used if two successor pairs $\langle$ stop, time $\rangle$ give an inconsistent speed due to errors in the data ${ }^{2}$.

\section{B. Multiagent System}

1) Planner Agents: The planner agents have the responsibility of computing the best itinerary for the traveler agents. A planner agent is created when an agent request is submitted to the system, and leaves the system right after. The planner agent bases its computation on the latest status of the networks. The graph representation and the paths calculations are described in section III-C. A road plan is composed of a sequence of edges together with their corresponding visit times. An itinerary is composed of a sequence of pairs $\left\langle i d_{\text {vehicle }}\right.$, itinerary $\rangle$, with $i d_{\text {vehicle }}$ the identifier of the vehicle to take and the corresponding part of the itinerary.

2) Agents Movements: Each active agent has a list of coordinates that he has to follow, resulting from the itinerary that he received from a planner agent. Agents are allowed to move for a certain distance at each tick equal to $\sigma_{P T \text { vehicle }}$ or $\sigma_{\text {passenger }}$ depending on the type of agent. At each tick, the agent iteratively checks if he can move from his current coordinate to the next in his list. If not, he calculates the intermediate coordinate that corresponds with the remaining distance that he is allowed to travel. In the next tick, the agent can travel the remaining distance to the next coordinate.

\footnotetext{
${ }^{2}$ E.g. infinite speed due to two identical visit times of two successive stops.
} 
3) Public Transport Vehicle Agents: The public transport vehicle agents don't choose their origin and destination and obey to predefined timetables. Each vehicle agent when created, infers his itinerary from his timetable. While the vehicle agent has not reached his destination, he travels at each tick the allowed distance, following his current speed. When the vehicle reaches a stop, he looks in his onboard travelers who has to leave at this stop. If there are passengers onboard, they are moved to the same coordinates at the same time by the vehicle agent. That means that, when they are onboard a a vehicle, traveler agents delegate the control of their movements to the vehicle agent. Then the vehicle agent looks in the list of waiting travelers at the stop who has to take him. At each simulation tick, the vehicle agent checks if he has reached his destination. If so, he leaves the simulation.

4) Traveler Agents: The origin and destination of the traveler agent are either inferred from travel patterns if they exist, or are chosen randomly. When they are not walking, traveler agents do not travel on their own, but share rides with other drivers and/or take public transport vehicles, which are responsible of their movements. The traveler agent alternates between walking and waiting for a vehicle.

\section{Itinerary Planning}

In order to avoid having passengers' shortest paths that pass by too many itineraries and thus result in too many transfers, we modify the public transport graph as follows (cf. Figure 2). Let $\left\langle s_{1}, s_{2}\right\rangle$ an edge in the public transport graph. Let $i t_{1}$ and $i t_{2}$ two itineraries passing by this edge. We create four new vertices $s_{1}^{\prime}, s_{2}^{\prime}$ that we connect with the edge that belongs to $i t_{1}$ and $s_{1}$ " and $s_{2}$ " that we connect with the edge that belongs to $i t_{2}$. We also create four new edges $\left\langle s_{1}^{\prime}, s_{1} "\right\rangle,\left\langle s_{1} ", s_{1}^{\prime}\right\rangle$, $\left\langle s_{2}^{\prime}, s_{2} "\right\rangle$ and $\left\langle s_{2} ", s_{2}^{\prime}\right\rangle$, that we note pedestrian edges and that are heavily penalized. When we run a shortest path algorithm on this new graph, the itineraries that are on the same vehicle/itinerary are encouraged and a transfer is only proposed when it is either impossible or really expensive to stay on the same vehicle.

After applying the A-Star shortest path algorithm on the resulting itinerary, the planner agent interrogates the stops of the best found itinerary to infer the sequence of vehicles that the traveler agent will have to take and sends back the result to the traveler agent.

\section{Traveler Information Management}

In this section, we describe the integration of traveler information into the SM4T simulator.

1) Local information Agents: Local information agents represent devices that broadcast trafic information on screens or voice announcement at the stops. With SM4T, it is possible to circumscribe the broadcast of messages to nearby traveler agents. Every traveler, when he passes

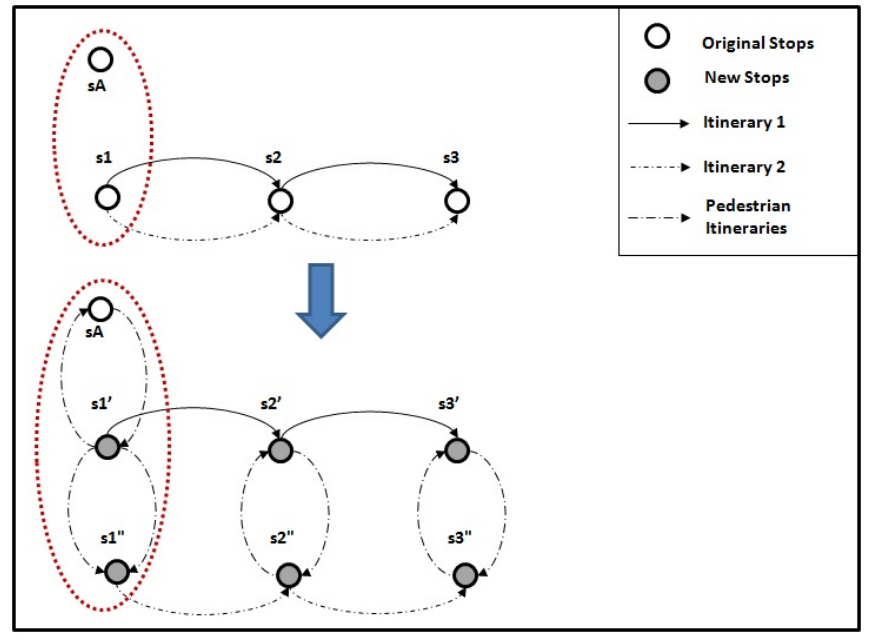

Fig. 2. Network Transformation

by a local information agent will get an update about the disturbances on the network (but no re-planning).

2) Travelers behaviors: Travelers that are not connected to a real-time information source base their calculation on a static view of the network. Once they have received an itinerary from the planner agent, they are on their own. They will wait for vehicles at the planned stops and will not change their itinerary until they either get stuck in a disturbance (delay or line disconnection) or they receive a local information about a disturbance. When they receive the information, they will infer the new status of the network by applying the modifications to their mental - and static - view of the transport network, and compute a shortest path based on that representation.

3) Connected Travelers behavior: Each connected traveler agent has his itinerary monitored. The monitoring purpose is to ask for a new itinerary in two cases:

1) the real position of the agent is different from the planned one with a certain gap $\Delta_{a}$ (defined in the preferences of the agent);

2) there is an event on the transport network that impacts the traveler itinerary.

The first case is handled by a behavior of the agents, since both the position of the agent and his plan are part of his knowledge. The second case however needs an interaction with the planner agent. Indeed, the planner monitors the itineraries of the travelers. To be aware of the only events that concern the agent, the planner subscribes the traveler to the only edges of the transport network that form his itinerary. When the travel time of an edge changes, the new travel time is broadcasted to the subscribing connected travelers. The planning process is then launched with the new travel times associated with the network.

\section{E. Injecting disturbances}

In order to validate the impact of real-time information, either at stops or personalized sent to individual 


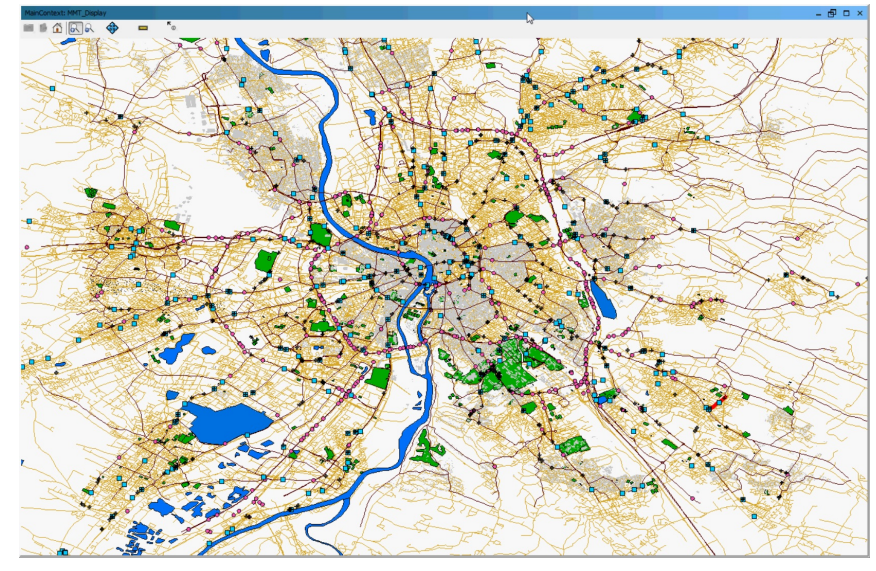

Fig. 3. Simulation Execution

travelers, we have to model disturbances. Indeed, it is in case of disturbances that information becomes critical in directing the passengers flows through the networks. Disturbances are modeled exclusively by modifying vehicles timetables. Indeed, delays are injected in the model by dynamically modifying the timetables of the vehicles, adding some time to arrival times. Breakdowns are modeled also by deleting a part of the mission of a vehicle. To model the breakdown of an entire line, the timetables of the remaining vehicles of the line are all deleted.

As soon as a timetable is modified, the information is broadcasted to all local information devices at stops. This is a simplification of the real situation, because in real systems, only a subset of information is broadcasted to the local information devices. Selecting which information has to be sent to which information device is an issue for the transport operator. In the scope of this paper, our objective is not to validate operators strategies, which would deserve a specific work on their own. Hence, we do not filter this information and broadcast it to all devices.

When a timetable is modified, based on the planner agent listeners, the information about the delay or the breakdown is sent to the only connected travelers that are interested by these vehicles missions.

\section{Experiments And Results}

\section{A. Setup}

We run our experiments on the city of Toulouse, France (cf. Figure 3), for which we have detailed data, including urban travel patterns [20]. We have considered the public transport network of Toulouse, with 80 lines, 359 itineraries and 3,887 arcs. In our current simulations, our multiagent system is made of 7,180 buses $^{3}$ and from 5,000 to 30,000 passengers. Travel times are updated with real data from the ClaireSiti platform [15]. We also have created random disturbances on the transit network (delays and missions deletion) to validate the impact of these events on the different travelers categories.

${ }^{3}$ In our simulations, we create one bus per mission.

\section{B. Scenarios}

We run six different scenarios, with four different numbers of traveler agents each. In the first scenario, no up-to-date information of any sort is given to the travelers. They only have the static description of the network and of the timetables. In the second scenario, information are given at some points of the networks. The information is only available for the passengers that are present in the concerned stations. No customer is connected in this scenario. The scenarios 3 to 6 represent the situations where local information is given to the travelers, and personal information is given to connected travelers: $20 \%, 50 \%, 80 \%$ and $100 \%$ of the travelers are connected, respectively. In each of these scenarios, we run 4 simulations with 5000, 10000, 20000 and 30000 passengers. For each of these 24 configurations, we report the mean travel times for the passengers.

\section{Results}

The results show that the scenario with no information provides the worst mean travel times. Indeed, in this scenario passengers will have the information about a delay or a breakdown when they are already stuck in a point of the network. While looking for an alternative, they will concurrently try to use the remainder of the network and might miss vehicles because of capacity constraints, and might also get stuck in another disturbance.

The scenario 2 (with exclusive local information) provides better results ( $23 \%$ in average) than scenario 1 . The positive impact of local information is due to the fact that the traveler does not have to arrive until the disturbance location to be informed, he will get it as soon as he passes by a station where information is broadcasted. However, he still can head towards another disturbance, since his itinerary is based on static information.

The integration of a certain percentage of connected passengers improves the average travel times until $50 \%$ of connected travelers. This time, the new itinerary of the travelers is calculated based on the real network status, and the passengers will avoid the disturbed zone quite early. He does not has to pass by a station where local information is provided, the passenger will receive a notification and a new plan quasi-immediately. The improvement of the scenario 3, with $20 \%$ connected passengers, compared to scenario 2 is of $27 \%$ in average. Scenario 4 , with $50 \%$ connected travelers provides better results than scenario 3 , with $15 \%$ improvement in the mean travel time.

Starting from scenario 5 , the results start to become worse. Indeed, with $80 \%$ of connected travelers for scenario 5 and $100 \%$ of connected travelers for scenario 6 , most of the travelers get personal real-time information about disturbances in real-time and new up-to-date plans are generated simultaneously. The consequence is that the travelers will face capacity constraints of the vehicles and will see their mean travel times increase. The results 


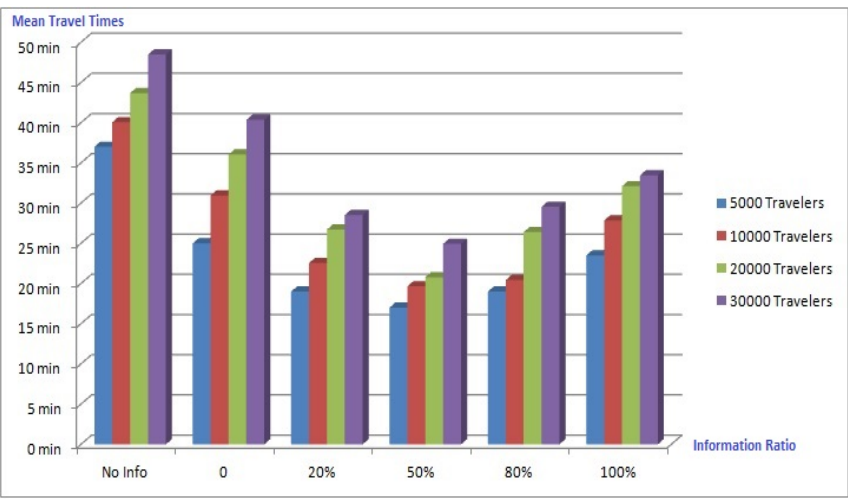

Fig. 4. Results

are $15 \%$ worse for scenario 5 compared to scenario 4 , and $23 \%$ worse for scenario 6 compared to scenario 5 .

The mean travel times increase w.r.t the number of travelers, whatever the scenario. The increase of the mean travel time is not very big, because we adapted the vehicles capacity to the number of considered travelers.

\section{Conclusion}

In this paper, we have described the main building blocks of a an agent-based simulator for transit travelers. We have presented the main functionalities allowing, among other applications, for the simulation of information exchange in the network. We presented a multiagent simulation model to measure the impact of information provision on the quality of passengers travels. This impact is measured by simulating different scenarios in function of the percentage of connected travelers, represented as agents. These simulated scenarios are analyzed following their impact on the average travel times of the travelers. Results show that the number of connected travelers has a positive impact on overall travel times up until a certain threshold before becoming negative.

In the near future, we plan to develop scenarios with hundreds of thousands of passengers to verify that our results are coherent with real volumes of data. The distribution of the simulator on the cloud is also currently conducted [12]. Our second ongoing research concerns the optimization of the distribution of connected travelers over competitive paths and not send them all to the same itineraries. Finally, we are extending the current work on multimodal networks.

\section{REFERENCES}

[1] A. L. Bazzan and F. Klügl. A review on agent-based technology for traffic and transportation. The Knowledge Engineering Review, 29(03):375-403, 2014.

[2] F. L. Bellifemine, G. Caire, and D. Greenwood. Developing Multi-Agent Systems with JADE. Wiley, 2007.

[3] M. Ben-Akiva, A. D. Palma, and K. Isam. Dynamic network models and driver information systems. Transportation Research Part A: General, 25(5):251 - 266, 1991.
4] N. Bessghaier, M. Zargayouna, and F. Balbo. Management of urban parking: an agent-based approach. In Artificial Intelligence: Methodology, Systems, and Applications, pages 276-285. Springer Berlin Heidelberg, 2012.

[5] S. Chipeaux, F. Bouquet, C. Lang, and N. Marilleau. Modelling of complex systems with AML as realized in MIRO project. In LAFLang 2011, workshop of the Int. Conf. WI/IAT (Web Intelligence and Intelligent Agent Technology), pages 159-162, Lyon, France, 2011. IEEE Computer Society.

[6] H. Dia. An agent-based approach to modelling driver route choice behaviour under the influence of real-time information rid b-1542-2008. Transportation Research Part C-emerging Technologies, 10(5-6):331-349, oct 2002 .

[7] J. Ferber and O. Gutknecht. Madkit: A generic multi-agent platform. In 4th International Conference on Autonomous Agents, pages 78-79, 2000.

[8] M. D. Hickman and N. H. Wilson. Passenger travel time and path choice implications of real-time transit information. Transportation Research Part C: Emerging Technologies, 3(4):211-226, 1995.

[9] M. Jakob, Z. Moler, A. Komenda, Z. Yin, A. X. Jiang, M. P. Johnson, M. Pechoucek, and M. Tambe. Agentpolis: towards a platform for fully agent-based modeling of multi-modal transportation (demonstration). In International Conference on Autonomous Agents and Multiagent Systems, AAMAS 2012, Valencia, Spain, June 4-8, 2012 (3 Volumes), pages 15011502, 2012.

[10] S. Luke, C. Cioffi-Revilla, L. Panait, and K. Sullivan. Mason: A new multi-agent simulation toolkit. In SwarmFest Workshop, 2004.

[11] M. Maciejewski and K. Nagel. Towards multi-agent simulation of the dynamic vehicle routing problem in matsim. In Proceedings of the 9th International Conference on Parallel Processing and Applied Mathematics - Volume Part II, PPAM'11, pages 551-560, Berlin, Heidelberg, 2012. Springer-Verlag.

[12] M. Mastio, M. Zargayouna, and O. Rana. Towards a distributed multiagent travel simulation. In Agent and Multi-Agent Systems: Technologies and Applications, page 10. Springer Berlin Heidelberg, 2015.

[13] N. Minar, R. Burkhart, C. Langton, and M. Askenazi. The swarm simulation system: A toolkit for building multi-agent simulations. Santa Fe Institute, 1996.

[14] K. Nagel and M. Rickert. Parallel implementation of the transims micro-simulation. Parallel Computing, 27(12):1611$1639,2001$.

[15] G. Scemama and O. Carles. Claire-siti, public road transport network management control: a unified approach. In Proc. of 12th IEE Int. Conf. on Road Transport Information \& Control (RTIC'04), pages 11-18, London (UK), April 2004.

[16] P. Taillandier, D.-A. Vo, E. Amouroux, and A. Drogoul. Gama: A simulation platform that integrates geographical information data, agent-based modeling and multi-scale control. In PRIMA, volume 7057 of Lecture Notes in Computer Science, pages 242-258. Springer, 2012.

[17] E. Tatara and J. Ozik. How to build an agent-based model iii - repast simphony. In Applied Agent-based Modeling in Management Research, Academy of Management Annual Meeting, Chicago, 2009.

[18] J. Wahle, A. L. C. Bazzan, F. KlÃijgl, and M. Schreckenberg. The impact of real-time information in a two-route scenario using agent-based simulation. Transportation Research Part C: Emerging Technologies, 10(5âĂS6):399 - 417, 2002.

[19] M. Zargayouna, B. Zeddini, G. SCEMAMA, and A. OTHMAN. Agent-based simulator for travelers multimodal mobility. Frontiers in Artificial Intelligence and Applications, 252:pp 81-90, Jan. 2013.

[20] M. Zargayouna, B. Zeddini, G. Scemama, and A. Othman. Simulating the impact of future internet on multimodal mobility. In The 11th ACS/IEEE International Conference on Computer Systems and Applications AICCSA'2014, page 10 pages. IEEE Computer Society, 2014. 